\title{
Rechtsgeschichte
}

\section{Jani Kirov}

\section{Unverrichteter Sache}


cherweise die typisch angelsächsische sprachliche Gefälligkeit und erzählerische Kraft erhalten geblieben sind, wird deshalb niemand vorbeikommen, der sich künftig mit irgendeinem Aspekt der deutschen Strafvollzugsgeschichte des Nationalsozialismus zu befassen beabsichtigt.

Thomas Krause

\section{Unverrichteter Sache*}

Das klassische Athen glänzte bereits in den Augen der Römer mit vielem, doch nicht mit seinem Recht. Die Athener übten sich in der Redekunst statt im Recht; statt Juristen hatten sie Redner und Redenschreiber. Für moderne Augen ein scheinbar diffiziler Fall: Wie, und warum auch, lässt sich hier Recht von anderen gesellschaftlichen Normen unterscheiden, wenn es keine Rechtskundigen gibt oder eher: wenn alle Bürger rechtskundig sind? Was heißt dann überhaupt Recht?

Solche Fragen irritieren Adriaan Lanni nicht. Sie kennt die Abneigung vieler Rechtshistoriker gegen das "amateurhafte Recht « der Griechen, und sie weiß auch, was Historiker daran meist interessiert: Willkür, Machtkämpfe, soziale und politische Funktionen. Lanni hingegen interessiert vornehmlich das Recht. Und die Gerechtigkeit. Das geht bereits aus dem Titel ihres Buches hervor: "Law and Justice in the Courts of Classical Athens ". Sie nimmt sich darin vor, Werte und Ideale des Rechts im klassischen Athen, das athenische Rechtsbewusstsein (»the Athenian legal mind «) zu erschließen. Natürlich nicht aus rechtstheoretischen oder rechtsdogmatischen Büchern, denn solche hatten und kannten die Athener nicht, sondern aus der Gerichtspraxis. Dazu bieten die überlieferten Gerichtsreden aus dem 5. und 4. Jahrhundert v. Chr., ferner Texte von Aristoteles und Plato sowie die klassische Dichtung reiches Material.

Dennoch kein einfaches Unterfangen. Denn selbst in actu war »the Athenian legal mind « nicht besonders offenbarungsfreudig. In den vielen Gerichtsreden ist nämlich kaum von Recht die Rede, und wenn, dann nicht in einer technischen Sprache. Oft wird weder ein Gesetz erwähnt noch »zur Sache« gesprochen. Man argumentierte für gewöhnlich ad hoc und ad personam: sei es die eigene oder die Person der Freunde und Feinde. Ebenso urteilten auch die Richter. Eigentlich urteilten sie gar nicht, sondern stimmten ab ( $\psi \eta \varphi i ́ \zeta \varepsilon \sigma \theta \alpha \imath)$.

Lanni will zeigen, dass das athenische Rechtssystem viel differenzierter und die Athener viel einsichtiger waren, als man üblicherweise annimmt. Während der Demokratie gab es nämlich parallel zu den Volksgerichten Gerichte für Klagen aus Mord und aus Seehandelsgeschäften. Dort habe eine Relevanzregel geherrscht, man habe also durchaus gewusst, was "zur Sache" gehöre und was nicht, und habe demzufolge sachlich, das heißt für Lanni: rechtlich argumentiert. Dieses inhomogene, weil aus unterschiedlichen Gerichtstypen bestehende Rechtssystem erkläre sich aus unterschiedlichen Rechts- und Gerechtigkeitsidealen, verdanke sich also nicht der blinden Evolution, sondern der Raison. Mit
Herausgebers) und 4I3-439 (Abdruck von Materialien) sowie Krause (Fn. 2), 85-88 m. w. N. (I3O-I3I).

I9 Beide soeben in Fn. I 8 angeführte Schriften werden von Wachsmann nicht erwähnt und erstaunlicherweise zitiert er auch nicht eine einzige Publikation des hannoverschen Strafrechtshistorikers Hinrich Rüping, obwohl dieser mehrere einschlägige Beiträge ver- fasst hat. In seiner englischsprachigen Einleitung findet sich denn auch ein kleiner Seitenhieb auf die Rechtsgeschichte (5), der allerdings interessanterweise in der deutschen Fassung fehlt.

\footnotetext{
* Adriaan Lanni, Law and Justice in the Courts of Classical Athens, Cambridge: Cambridge University Press 2006, 2 10 S., ISBN-I 3 978-0-52 I-8 5759-8; ISBN-IO O-52 I-8 5759-7
} 
der Errichtung der Volksgerichte hätten sich die Athener bewusst für ein demokratisches Rechtsund Gerechtigkeitsideal entschieden, ohne das alte, »formalistische " gänzlich $\mathrm{zu}$ verwerfen. Dadurch hätten sie auch das »ewige Problem « gelöst, auf welches jede Gesellschaft beim Aufbau ihres Rechtssystems stoße: »the tension between adherence to general rules and doing justice in specific cases « (I75).

Die Verfasserin glaubt damit auch die heute weitgehend akzeptierte Auffassung widerlegen zu können, wonach der Rechtsstreit in den athenischen Gerichten einen Wettstreit um Ehre und Prestige bedeutete. Dies sei, so Lanni, eine Ausnahme gewesen, kein Ideal. Wenn die Athener den Unterschied zwischen formaler und informaler Gerechtigkeit erkannt und verwirklicht hätten, dann seien sie auch dazu fähig gewesen, rechtliche und außerrechtliche Zwecke auseinander zu halten. Dann hätten die Gerichte allein rechtlichen Zwecken, dem Recht und der Gerechtigkeit gedient, mag die vor Gericht zugelassene Argumentation selbst auch außerrechtlicher Natur gewesen sein. Diese Schlussfolgerung sieht die Verfasserin im Einklang auch mit der neuen Moral der athenischen Gesellschaft im 5. Jahrhundert v. Chr. Damals sei das Individuum als moralisch verantwortlich begriffen worden. Die kompetitive Ethik der alten Zeit sei durch eine demokratische verdrängt worden, die auf Selbstenthaltung, Kooperation und Gewissen gegründet habe. Kurz: statt $\pi 0 \lambda \lambda$ òv

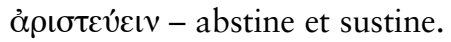

Thesen dieser Art laufen oft Ergebnissen der modernen Gräzistik entgegen, die mehr durch die Moral übertönt als wissenschaftlich widerlegt werden. Dies führt öfter dazu, dass auch wissenschaftliche Annahmen, die die Autorin selbst aufgestellt hat, aus dem Blick geraten und zusammenhanglos bleiben. So wird etwa der wichtige Hinweis auf die während der Demokratie mitnichten überwundene starke soziale Differenzierung innerhalb der athenischen Gesellschaft (20-2I) nicht weiter verfolgt. Damit wird aber die soziale Bedingtheit und Funktionalität nicht allein der moralischen Werte und Ideale ausgeblendet, auf die es Lanni ankommt, sondern auch jedweder historischen Selbstbeschreibung. Statt die Behauptungen der Prozessparteien zu hinterfragen, gehen Lannis Bemühungen dahin, ihnen Glaubwürdigkeit ab- oder zuzusprechen.

So bleibt einem nur zu glauben, dass die Athener glaubten, woran Lanni glaubt: dass Moral und Recht menschliches Handeln regieren; dass soziale Institutionen aus Idealen und Bedürfnissen der Gesellschaft entstehen; dass Recht in der Moral begründet ist. Und die Gerechtigkeit? Was ist sie? Eine Tugend, eine Idee, ein moralischer Wert, eine Norm neben dem Recht oder gar oberhalb des Rechts? Aber gibt es noch Recht, wenn moralische Normen als Selektionskriterien des Rechts fungieren - »so als ob es neben dem Baurecht und dem Straßenverkehrsrecht, dem Erbrecht und dem Urheberrecht auch noch gerechtes Recht gäbe «? ${ }^{\mathrm{I}}$ Und schließlich: Wer zieht hier die Grenze zwischen Recht und Nichtrecht? Die Gesellschaft? Aber wer ist das? Und meinte der durchschnittliche Athener mit Wendungen wie »zur Sache« bzw.

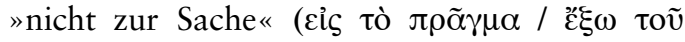
$\pi \rho \alpha ́ \gamma \mu \alpha \tau o \varsigma)$ das, was Lanni mit Recht/Nichtrecht meint? Und was eigentlich meint sie damit? Lauter Fragen ohne Antworten. Und lauter Antworten ohne Fragen, Antworten nicht zur Sache.

I Niklas Luhmann, Das Recht der Gesellschaft, Frankfurt a. M.

I993, 216. 